\title{
Polychlorinated biphenyls in sediments and fish species from the Murchison Bay of Lake Victoria, Uganda
}

\author{
Patrick Ssebugere ${ }^{\mathrm{a}, \mathrm{c}}$, Mika Sillanpää ${ }^{\mathrm{b}}$, Pu Wang ${ }^{\mathrm{c}}$, Yingming Li $^{\mathrm{c}}$, Bernard T. Kiremire ${ }^{\mathrm{a}}$, Gabriel N. Kasozi ${ }^{\mathrm{a}}$, \\ Chaofei Zhu ${ }^{c}$, Daiwei Ren ${ }^{c}$, Nali Zhu ${ }^{c}$, Haidong Zhang ${ }^{c}$, Hongtao Shang ${ }^{c}$, Qinghua Zhang ${ }^{c}, *$, Guibin Jiang ${ }^{c}$ \\ a Department of Chemistry, Makerere University, P.O. Box 7062, Kampala, Uganda \\ b Laboratory of Green Chemistry, Lappeenranta University of Technology, Sammonkatu 12, 50130 Mikkeli, Finland \\ c State Key Laboratory of Environmental Chemistry and Ecotoxicology, Research Center for Eco-Environmental Sciences, Chinese Academy of Sciences, Beijing 100085, China
}

\section{H I G H L I G H T S}

- High concentrations of PCBs were found at sites near wastewater discharges.

- The PCB concentrations were low to moderate compared to other locations worldwide.

- Based on the European Commission set TEQ the fish were fit for human consumption.

\section{A R T I C L E I N F O}

Article history:

Received 12 December 2013

Received in revised form 2 March 2014

Accepted 2 March 2014

Available online 21 March 2014

\section{Keywords:}

Polychlorinated biphenyls

Sediments

Fish

Murchison Bay

Lake Victoria

\begin{abstract}
A B S T R A C T
Polychlorinated biphenyls (PCBs) were determined in sediments and two fish species collected from the Murchison Bay in Lake Victoria, using high resolution gas chromatography coupled to a high resolution mass spectrometer. Total PCB concentrations ( $\Sigma_{18} \mathrm{PCBs}$ ) varied widely with mean values ranging from 777 to $4325 \mathrm{pg} \mathrm{g}^{-1}$ dry weight ( $\mathrm{dw}$ ) for sediments and 80 to $779 \mathrm{pg} \mathrm{g}^{-1}$ wet weight (ww) for fish. The PCB levels in the sediments were significantly higher at the station closest to Nakivubo channel, presumably due to effluents discharged by the channel, which may contain domestically produced commercial PCB mixtures. For fish, the concentrations in Nile perch (Lates niloticus) were significantly greater than those in Nile tilapia (Oreochromis niloticus) at all study stations, possibly due to dietary differences among species. World Health Organization-toxic equivalents $\left(\mathrm{WHO}_{2005}\right.$-TEQs) for the dioxin-like PCBs were 0.04-0.64 $\mathrm{pg} \mathrm{g}^{-1} \mathrm{dw}$ and $0.01-0.39 \mathrm{pg} \mathrm{g}^{-1} \mathrm{ww}$ for sediments and fish, respectively. The non-ortho PCBs exhibited the highest contribution to the $\Sigma_{12} \mathrm{TEQs}(>75 \%)$ compared to the mono-ortho PCBs in both fish species. The TEQs in the present study were lower than many reported worldwide in literature for fish and were within the permissible level recommended by the European Commission, implying that the fish did not pose health hazards related to PCBs to the consumers.
\end{abstract}

(C) 2014 Elsevier B.V. All rights reserved.

\section{Introduction}

Lake Victoria is a vital natural aquatic resource in Uganda, but its ecological health is being threatened, mainly by rapid urbanization and industrialization. These developments coupled with a population of over one million people in its catchment area have resulted in increased pollution (Banadda et al., 2009, 2011a, 2011b; Wasswa et al., 2010). Polychlorinated biphenyls (PCBs) have previously been documented in the lake (Ssebugere et al. 2013a, b; Ssebugere et al., 2014). The PCBs are released into the environment as a result of industrial applications such as capacitors, hydraulic fluids in electronic

\footnotetext{
* Corresponding author. Tel.: + 8613693263173.

E-mail address: qhzhang@rcees.ac.cn (Q. Zhang).
}

equipment, and coolants and lubricants in transformers (Gidarakos et al., 2009; Moon et al., 2012; Lundin et al., 2013; Miller et al., 2013).

Exposure to PCBs has been associated with diseases like diabetes mellitus, cancer and hypertension (Lee et al., 2007a). Other adverse effects caused by the pollutants include endocrine disruption, neurobehavioral deficits and fetal toxicity (Lee et al., 2007b; Ha et al., 2007; 2009; Uemura et al., 2009). The PCBs undergo long-range transport causing global pollution (Wiberg et al., 2009; Sundqvist et al., 2009a, b). Furthermore, the contaminants can accumulate in sediments close to point sources and in benthic organisms and fish due to their hydrophobic nature, low metabolic transformation and long half-lives (Suedel et al., 1994; Jönsson et al., 2003; Åberg et al., 2008; 2010; Lavandier et al., 2013). Fish can be an important monitoring tool, because they concentrate PCBs directly from water through their diet, and indicate hazards to piscivorous predators and humans (Van 
der Oost et al., 2003; Huang et al., 2006; Gustavson et al., 2008; Parnell et al., 2008).

To our knowledge whereas PCBs have been reported in fish and sediments from the Napoleon Gulf and Thurston Bay of Lake Victoria (Ssebugere et al., 2013a, b; Ssebugere et al., 2014) and from other water bodies in the world (Manirakiza et al., 2002; Adu-Kumi et al., 2010; Darko et al., 2008; Helm et al., 2008; Eqani et al., 2013; Verhaert et al., 2013), literature shows no work about these pollutants in the Murchison Bay. The Bay is a major hotspot for discharge of industrial outflow, urban runoff and municipal effluents from Kampala, Uganda's capital city (Banadda et al., 2009). The objectives of this study were to investigate the occurrence and contamination levels of PCBs in sediments and fish from the Murchison Bay.

\section{Materials and methods}

\subsection{Study area}

The area of study was the Murchison Bay located in the northwestern part of Lake Victoria and southeast of Kampala city (Fig. 1). The Bay covers an area of $60 \mathrm{~km}^{2}$ and includes semi-enclosed inner and outer parts. The Murchison Bay is the main recipient of sewage effluents, industrial- and municipal-waste from the central district of Kampala City, via the Nakivubo channel. The channel passes through dense residential settlements and commercial areas. In the past, the channel ended in wetland areas, allowing wastewater to be drained into papyrus swamps before entering the Bay. However, in recent years the wetlands which played the role of secondary treatment have been degraded due to settlement and commercial activities (Wasswa, 2009). The water turbidity in the Bay has increased while the water transparency has declined due to excessive waste input.

\subsection{Sample collection}

A total of 24 surface sediments ( $<25 \mathrm{~cm}$ depth) were taken using a sediment corer from four stations namely 1 and 2 in the inner Bay, and 3 and 4 in the outer Bay (Fig. 1) in March, 2013. Six sediments were randomly taken from each station at distances of approximately
$200 \mathrm{~m}$ from one another. The geographical coordinates of the inner and outer parts of the Murchison Bay are $00^{\circ} 15^{\prime} 72^{\prime \prime} \mathrm{N}, 32^{\circ} 38^{\prime} 74^{\prime \prime} \mathrm{E}$ and $00^{\circ} 08^{\prime} 71^{\prime \prime} \mathrm{N}, 32^{\circ} 37^{\prime} 58^{\prime \prime} \mathrm{E}$, respectively. Ninety six fish of different species namely: Nile Perch (Lates niloticus) a carnivorous top-predator and Nile Tilapia (Oreochromis niloticus) a detritivorous species were collected using gill nets within the same locality as the sediments. These two species exhibit different feeding habitats and thus may be exposed differently to contamination through trophic transfer. The weights of O. niloticus ranged from 289 to $652 \mathrm{~g}$ (mean $389 \mathrm{~g}$ ) while those of L. niloticus varied from 1628 to $2979 \mathrm{~g}$ (mean $2378 \mathrm{~g}$ ). The mean lengths ( \pm standard deviation) were $29 \pm 3 \mathrm{~cm}$, and $68 \pm 5 \mathrm{~cm}$, respectively. The muscle tissues were dissected between the pectoral fin and vent of the fish, minced into pieces and subsamples taken. Subsamples of 4 fish from the same location and, of similar length and species were pooled and homogenized. The sediments and homogenized fish were transferred into acetone rinsed glass bottles and shipped to the Research Center for Eco-Environmental Sciences, Chinese Academy of Sciences where they were freeze-dried.

\subsection{Analytical method}

Sample extraction and cleanup procedures followed the method reported by Wang et al. (2013). Briefly, $2 \mathrm{~g}$ of the freeze-dried sample (fish or sediment) was ground with $10 \mathrm{~g}$ of anhydrous sodium sulfate to a free floating powder using a mortar and pestle. The mixture was spiked with ${ }^{13} \mathrm{C}$-labeled surrogate standards (US EPA defined 68A-LCS) and extracted on an accelerated solvent extractor device (ASE 300, Dionex, USA) using 1:1 v/v mixture of $n$-hexane/dichloromethane (DCM). The resultant extract was concentrated to $2 \mathrm{~mL}$ on a rotary evaporator. The concentrated extract was divided into two subsamples for fish $(0.3$ and $1.7 \mathrm{~mL}$ ). The $0.3 \mathrm{~mL}$ was used for gravimetric determination of the lipid content while the $1.7 \mathrm{~mL}$ was kept for clean-up. For the sediments, activated copper granules were added to the extract to remove elemental sulfur before purification with column chromatography. The extracts were then cleaned up with a multilayered silica column (packed from bottom to top with $1 \mathrm{~g}$ silica gel, $4 \mathrm{~g}$ basic silica gel, $1 \mathrm{~g}$ silica gel, $8 \mathrm{~g}$ acid silica gel, $2 \mathrm{~g}$ silica gel and $2 \mathrm{~g}$ anhydrous sodium sulfate), an alumina column ( $6 \mathrm{~g}$ basic alumina and $3 \mathrm{~g}$ anhydrous sodium sulfate

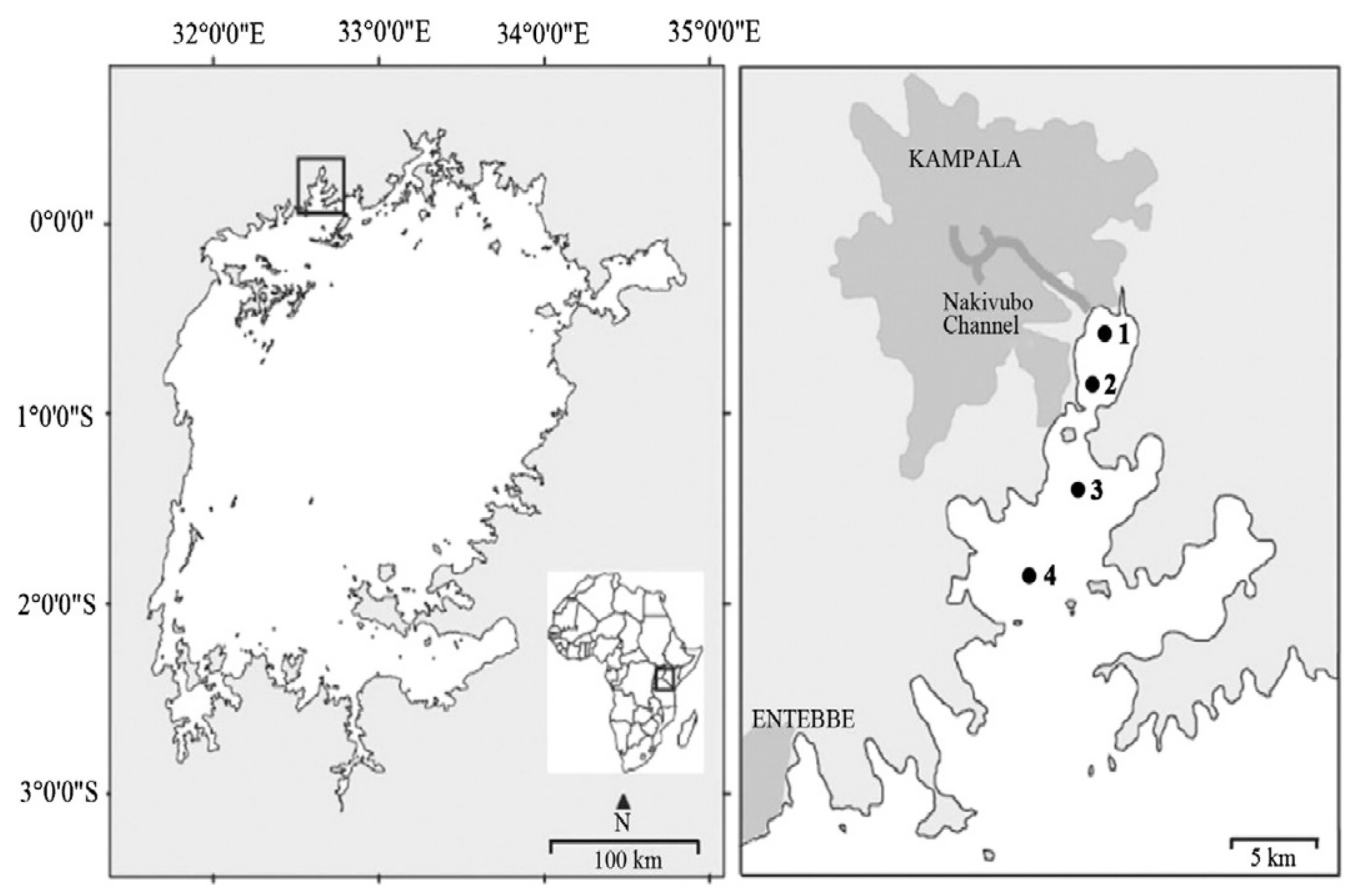

Fig. 1. Map of the Murchison Bay showing the study stations. Adapted from Haande et al. (2011). 
Table 1

Mean concentrations and ranges ( $\mathrm{pg} \mathrm{g}^{-1}$ dry weight) of PCBs in sediments from the Murchison Bay.

\begin{tabular}{|c|c|c|c|c|}
\hline Congener & Station 1 & Station 2 & Station 3 & Station 4 \\
\hline CB 28 & $\begin{array}{l}375 \pm 25 \\
353-402\end{array}$ & $\begin{array}{l}318 \pm 30 \\
284-340\end{array}$ & $\begin{array}{l}103 \pm 54 \\
51-158\end{array}$ & $\begin{array}{l}63 \pm 21 \\
45-85\end{array}$ \\
\hline CB 52 & $\begin{array}{l}507 \pm 99 \\
397-589\end{array}$ & $\begin{array}{l}293 \pm 31 \\
259-319\end{array}$ & $\begin{array}{l}169 \pm 14 \\
153-179\end{array}$ & $\begin{array}{l}108 \pm 44 \\
58-137\end{array}$ \\
\hline CB 101 & $\begin{array}{l}585 \pm 234 \\
420-853\end{array}$ & $\begin{array}{l}343 \pm 176 \\
229-546\end{array}$ & $\begin{array}{l}158 \pm 68 \\
105-235\end{array}$ & $\begin{array}{l}122 \pm 41 \\
75-150\end{array}$ \\
\hline CВ 138 & $\begin{array}{l}933 \pm 446 \\
631-1445\end{array}$ & $\begin{array}{l}553 \pm 396 \\
230-1009\end{array}$ & $\begin{array}{l}240 \pm 131 \\
151-390\end{array}$ & $\begin{array}{l}153 \pm 87 \\
79-249\end{array}$ \\
\hline CВ 153 & $\begin{array}{l}898 \pm 240 \\
721-1171\end{array}$ & $\begin{array}{l}585 \pm 465 \\
251-1115\end{array}$ & $\begin{array}{l}227 \pm 90 \\
173-331\end{array}$ & $\begin{array}{l}177 \pm 85 \\
110-272\end{array}$ \\
\hline CB 180 & $\begin{array}{l}218 \pm 63 \\
152-276\end{array}$ & $\begin{array}{l}117 \pm 54 \\
61-169\end{array}$ & $\begin{array}{l}49 \pm 23 \\
26-72\end{array}$ & $\begin{array}{l}29 \pm 6.1 \\
23-35\end{array}$ \\
\hline$\Sigma$ indicator-PCBs & $\begin{array}{l}3516 \\
2749-4652\end{array}$ & $\begin{array}{l}2209 \\
1464-3488\end{array}$ & $\begin{array}{l}946 \\
719-1282\end{array}$ & $\begin{array}{l}652 \\
409-921\end{array}$ \\
\hline CB 77 & $\begin{array}{l}82 \pm 20 \\
60-99\end{array}$ & $\begin{array}{l}56 \pm 6.3 \\
52-64\end{array}$ & $\begin{array}{l}12 \pm 4.5 \\
6.3-14\end{array}$ & $\begin{array}{l}8.8 \pm 1.2 \\
8.1-10\end{array}$ \\
\hline CB 81 & $\begin{array}{l}1.9 \pm 1.1 \\
0.7-2.8\end{array}$ & $\begin{array}{l}2.5 \pm 0.9 \\
1.6-3.3\end{array}$ & $\begin{array}{l}1.0 \pm 0.6 \\
0.6-1.4\end{array}$ & $\begin{array}{l}0.8 \pm 0.3 \\
0.6-1.0\end{array}$ \\
\hline CB 126 & $\begin{array}{l}4.9 \pm 1.4 \\
3.3-5.8\end{array}$ & $\begin{array}{l}2.2 \pm 0.7 \\
1.7-3.0\end{array}$ & $\begin{array}{l}1.2 \\
\text { n.d.-1.2 }\end{array}$ & $\begin{array}{l}0.9 \pm 0.5 \\
\text { n.d.-1.2 }\end{array}$ \\
\hline CВ 169 & n.d. & $\begin{array}{l}0.3 \\
\text { n.d. }-0.3\end{array}$ & n.d. & $\begin{array}{l}0.1 \\
\text { n.d.-0.1 }\end{array}$ \\
\hline$\Sigma$ non-ortho-PCBs & $\begin{array}{l}89 \\
66-105\end{array}$ & $\begin{array}{l}61 \\
55-70\end{array}$ & $\begin{array}{l}14 \\
6.8-17\end{array}$ & $\begin{array}{l}11 \pm 2.0 \\
8.9-13\end{array}$ \\
\hline CB 105 & $\begin{array}{l}140 \pm 70 \\
99-221\end{array}$ & $\begin{array}{l}59 \pm 18 \\
47-79\end{array}$ & $\begin{array}{l}26 \pm 10 \\
19-38\end{array}$ & $\begin{array}{l}22 \pm 13 \\
11-36\end{array}$ \\
\hline CB 114 & $\begin{array}{l}6.7 \pm 3.3 \\
4.4-11\end{array}$ & $\begin{array}{l}2.9 \pm 0.6 \\
2.3-3.4\end{array}$ & $\begin{array}{l}1.9 \pm 0.6 \\
1.5-2.6\end{array}$ & $\begin{array}{l}1.2 \pm 0.6 \\
0.7-1.9\end{array}$ \\
\hline CB 118 & $\begin{array}{l}434 \pm 214 \\
302-682\end{array}$ & $\begin{array}{l}174 \pm 74 \\
139-228\end{array}$ & $\begin{array}{l}110 \pm 59 \\
71-177\end{array}$ & $\begin{array}{l}72 \pm 45 \\
32-120\end{array}$ \\
\hline CB 123 & $\begin{array}{l}53 \pm 19 \\
42-76\end{array}$ & $\begin{array}{l}24 \pm 7.4 \\
18-32\end{array}$ & $\begin{array}{l}13 \pm 5.3 \\
8.7-19\end{array}$ & $\begin{array}{l}7.4 \pm 6.2 \\
1.0-13\end{array}$ \\
\hline CB 156 & $\begin{array}{l}54 \pm 28 \\
36-86\end{array}$ & $\begin{array}{l}28 \pm 14 \\
16-43\end{array}$ & $\begin{array}{l}14 \pm 5.1 \\
9.2-19\end{array}$ & $\begin{array}{l}7.5 \pm 3.3 \\
4.9-11\end{array}$ \\
\hline CВ 157 & $\begin{array}{l}7.7 \pm 5.2 \\
4.2-14\end{array}$ & $\begin{array}{l}3.4 \pm 1.3 \\
2.2-4.8\end{array}$ & $\begin{array}{l}2.0 \pm 1.0 \\
1.3-3.1\end{array}$ & $\begin{array}{l}1.3 \pm 0.7 \\
0.8-2.1\end{array}$ \\
\hline CB 167 & $\begin{array}{l}16 \pm 7.9 \\
11-26\end{array}$ & $\begin{array}{l}8.9 \pm 5.0 \\
4.6-14\end{array}$ & $\begin{array}{l}4.4 \pm 1.1 \\
3.1-5.2\end{array}$ & $\begin{array}{l}1.9 \pm 0.4 \\
1.5-2.2\end{array}$ \\
\hline CB 189 & $\begin{array}{l}8.0 \pm 1.9 \\
6.6-10\end{array}$ & $\begin{array}{l}5.5 \pm 2.7 \\
3.0-8.4\end{array}$ & $\begin{array}{l}2.7 \pm 1.3 \\
1.3-3.9\end{array}$ & $\begin{array}{l}1.1 \pm 0.4 \\
0.8-1.6\end{array}$ \\
\hline$\Sigma$ mono-ortho-PCBs & $\begin{array}{l}720 \\
508-1124\end{array}$ & $\begin{array}{l}306 \\
231-413\end{array}$ & $\begin{array}{l}174 \\
124-266\end{array}$ & $\begin{array}{l}114 \\
53-189\end{array}$ \\
\hline$\Sigma_{12} \mathrm{dl}-\mathrm{PCBs}$ & $\begin{array}{l}809 \\
613-1190\end{array}$ & $\begin{array}{l}367 \\
289-482\end{array}$ & $\begin{array}{l}188 \\
131-283\end{array}$ & $\begin{array}{l}125 \\
62-202\end{array}$ \\
\hline$\Sigma_{18} \mathrm{PCBs}$ & $\begin{array}{l}4325 \\
3762-5842\end{array}$ & $\begin{array}{l}2576 \\
1753-3970\end{array}$ & $\begin{array}{l}1134 \\
850-1365\end{array}$ & $\begin{array}{l}777 \\
471-1123\end{array}$ \\
\hline $\mathrm{WHO}_{2005}$-TEQs & $\begin{array}{l}0.55 \\
0.37-0.64\end{array}$ & $\begin{array}{l}0.25 \\
0.19-0.33\end{array}$ & $\begin{array}{l}0.11 \\
0.04-0.12\end{array}$ & $\begin{array}{l}0.10 \\
0.07-0.14\end{array}$ \\
\hline
\end{tabular}

The number of sediment samples per station $(\mathrm{N})=6$; n.d. - non-detectable. at the top) and carbon column ( $1.5 \mathrm{~g}$ of $18 \%$ carbon dispersed in cilite, $3 \mathrm{~g}$ anhydrous sodium sulfate at the top), respectively. The clean eluate was concentrated to $1 \mathrm{~mL}$ using a rotary evaporator and transferred into vials containing $20 \mu \mathrm{L}$ nonane. The contents in the vials were further dried to $25 \mu \mathrm{L}$ using a gentle stream of nitrogen and later spiked with injection standards (US EPA defined 68A-IS) prior to analysis.

\subsection{Instrumental analysis}

The analysis of 18 PCB congeners including 6 indicator PCBs (IUPAC numbers 28, 52, 101, 138, 153 and 180) and twelve dioxin-like PCBs (IUPAC numbers 77, 81, 126, 169, 105, 114, 118, 123, 156, 157, 167 and 189) was done using high-resolution gas chromatography coupled with high-resolution mass spectrometry (HRGC/HRMS) (AutoSpec Ultima, Waters, USA). Chromatographic separation was achieved by injecting $1 \mu \mathrm{L}$ of sample on a fused silica capillary column (DB5MS, $60 \mathrm{~m} \times 0.25 \mathrm{~mm}$ i.d. $\times 0.25 \mu \mathrm{m}$ film thicknesses). The oven temperature of the GC was held at $120{ }^{\circ} \mathrm{C}$ for $1 \mathrm{~min}$, then increased to $150{ }^{\circ} \mathrm{C}$ at a rate of $30{ }^{\circ} \mathrm{C} \mathrm{min}-1$ and finally to $300{ }^{\circ} \mathrm{C}$ at $2.5^{\circ} \mathrm{C} \mathrm{min}^{-1}$. Helium was used as a carrier gas at a flow rate of $1.0 \mathrm{~mL} \mathrm{~min}^{-1}$. The HRMS was operated in VSIR mode at a resolution of $\geq 10000$ under positive electron ionization conditions $(35 \mathrm{eV})$ and a source temperature of $270{ }^{\circ} \mathrm{C}$.

\subsection{Quality assurance and quality control}

To ensure the quality of data, surrogate PCB standards, blanks, replicates and a certified reference material (CRM) were included in the analysis. Recoveries for majority of the surrogate standards met the requirements of US EPA methods 1668A (were in the range of 75-127\%) except for CBs 28,118 , and 138 whose recoveries were $<75 \%$ (range: $56-74 \%$ ). The recoveries resulting from triplicate determinations $(\mathrm{N}=3)$ of the certified reference material were between $82 \%$ and $106 \%$. The limit of detection (LOD) which was calculated as three times the signal-to-noise ratio, varied from 0.04 to $0.96 \mathrm{pg} \mathrm{g}^{-1}$ dry weight (dw) for sediments and 0.02 to $0.80 \mathrm{pg} \mathrm{g}^{-1}$ wet weight (ww) for fish. PCBs in the field and procedural blanks were detected at $<15 \%$ of the concentration in each batch of 12 samples, so the reported data were not blank corrected.

\subsection{Statistical data analysis}

Statistical analysis was performed using Statistica ${ }^{\circledR} 7$ for Windows. Kolmogorov-Smirnov test was used for verifying the normality of data. Since concentration values were not normally distributed, nonparametric statistics was applied. The Mann-Whitney $U$-test was used for pair wise comparison and the Kruskal-Wallis test when more than two groups were considered. In these two non-parametric tests, statistical significance was set at $p<0.05$. In the present study, toxic

Table 2

Comparison of sediment PCB concentrations ( $\mathrm{pg} \mathrm{g}^{-1}$ dry weight) in this study with those from other locations.

\begin{tabular}{|c|c|c|}
\hline Area & PCBs (pg g ${ }^{-1}$ dry weight) & References \\
\hline Napoleon Gulf and Thurston Bay of Lake Victoria, Uganda & 64-1262 (18 congeners) & Ssebugere et al. (2013b) and Ssebugere et al. (2014) \\
\hline Nile River, Egypt & 1461-2244 (18 congeners) & El-Kady et al. (2007) \\
\hline Pangani River basin, Tanzania & $357-11000$ (28 congeners) & Hellar-Kihampa et al. (2013) \\
\hline Congo River basin, Democratic Republic of Congo & n.d.-1400 (33 congeners) & Verhaert et al. (2013) \\
\hline Ghar El Melh lagoon, Tunisia & n.d.-3987 (20 congeners) & Ameur et al. (2011) \\
\hline Mekong River delta, Vietnam & $110-2000$ (13 congeners) & Carvalho et al. (2008) \\
\hline Riverine and coastal waters of Surabaya, Indonesia & n.d. -420000 (62 congeners) & Ilyas et al. (2011) \\
\hline Scheldt River, Belgium & n.d.-200000 (27 congeners) & Covaci et al. (2005) \\
\hline Remote lakes and coastal areas, Norwegian Arctic & $180-13000$ (15 congeners) & Jiao et al. (2009) \\
\hline Mersey Estuary, United Kingdom & $36000-1409000$ (7 congeners) & Vane et al. (2007) \\
\hline Lake Maggiore, Italy and Switzerland & n.d. -3000 (12 congeners) & Vives et al. (2007) \\
\hline Besòs River, Barcelona & $6-14(30$ congeners $)$ & Castells et al. (2008) \\
\hline Indiana Harbor and Ship Canal, Lake Michigan, United States of America & $53000-35000000$ (163 congeners) & Martinez et al. (2010) \\
\hline Sea Lots, Port of Spain, Trinidad and Tobago & 62000-601000 (136 congeners) & Mohammed et al. (2011) \\
\hline
\end{tabular}

n.d. - non-detectable. 


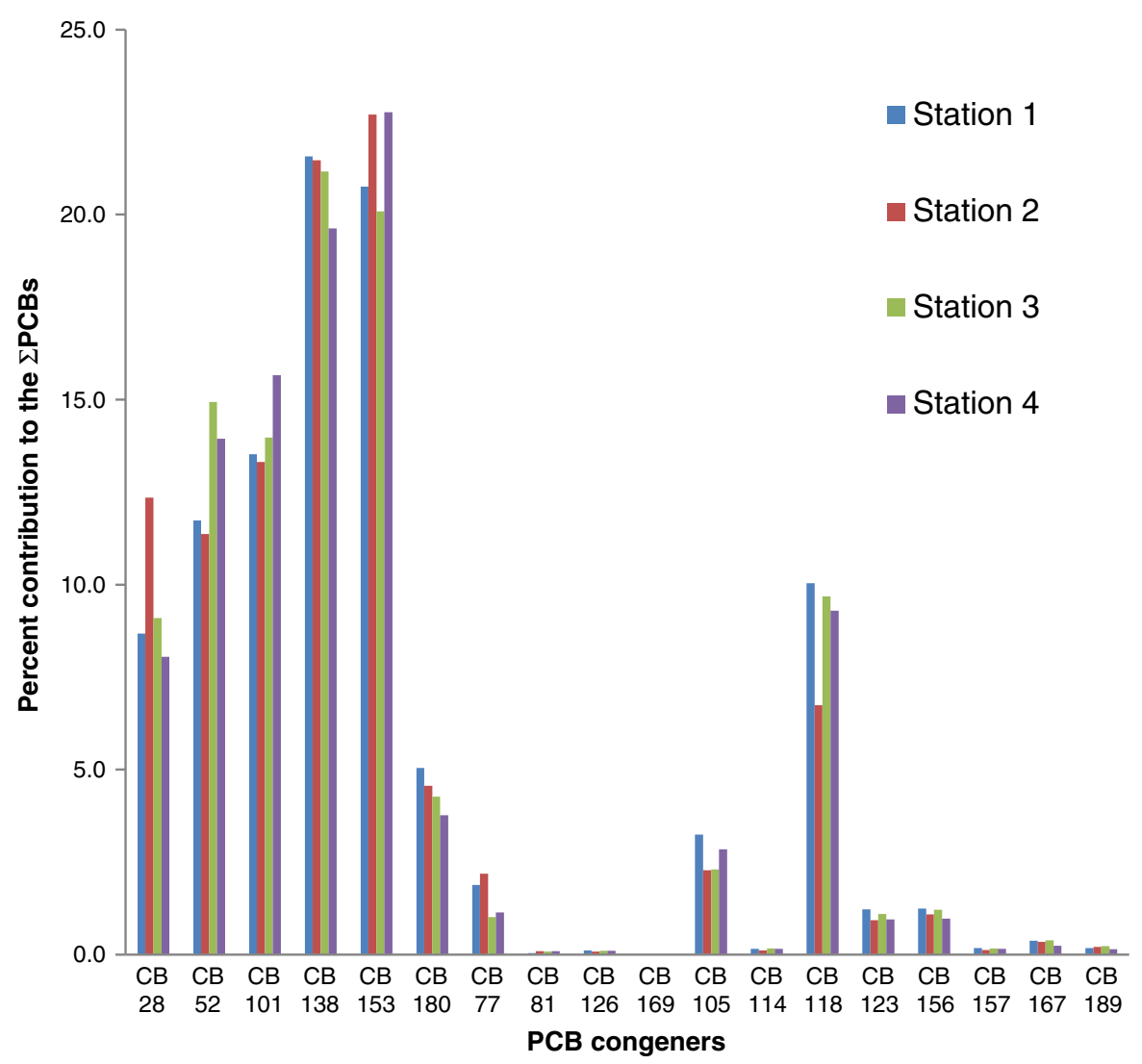

Fig. 2. Percent contribution of the congeners to the $\Sigma_{18} \mathrm{PCBs}$ per station.

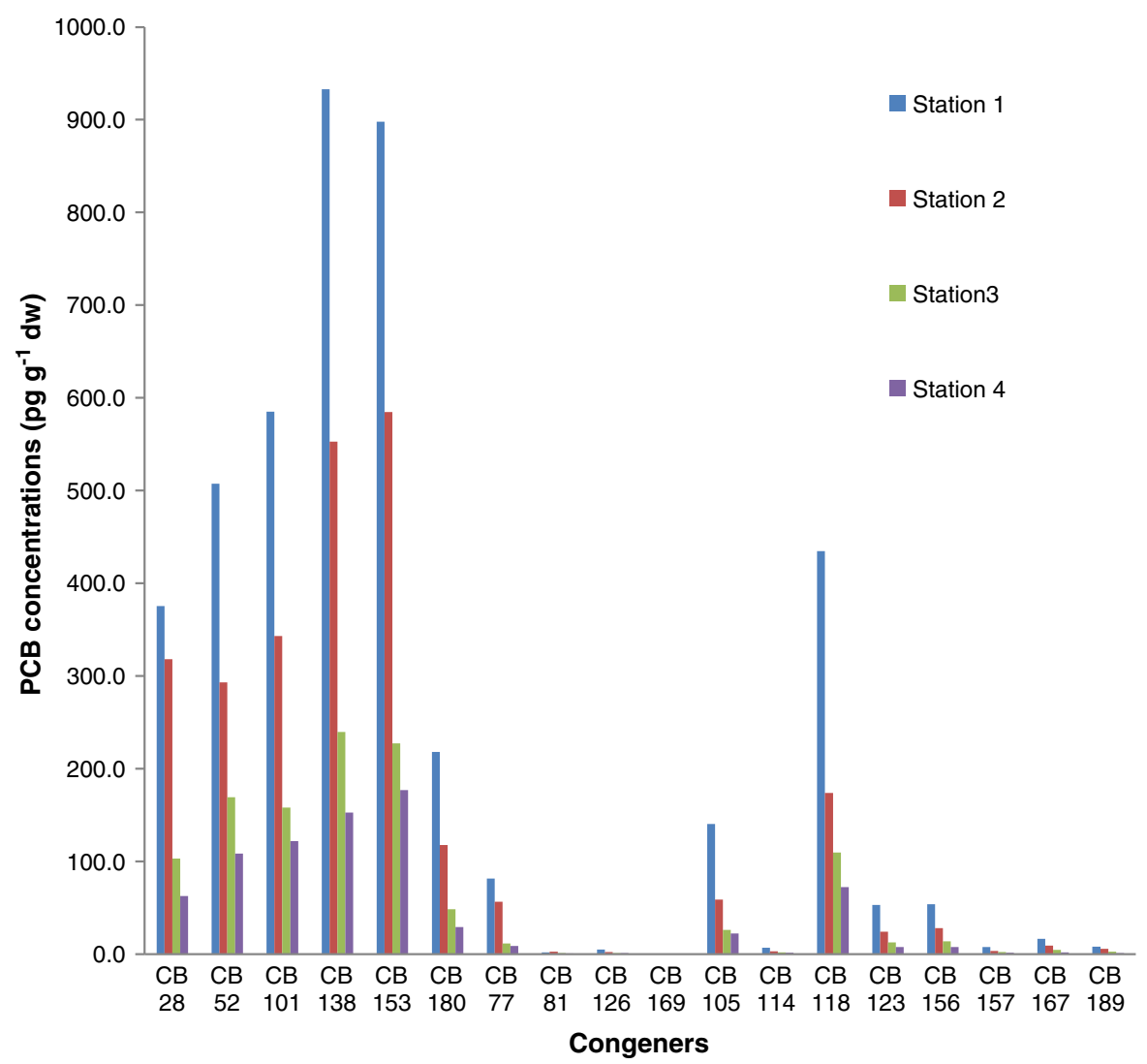

Fig. 3. Mean concentrations of the $\mathrm{PCB}$ congeners in the sediments per station. 
equivalents (TEQs) for the 12 dioxin-like (dl) PCBs were calculated using individual congener concentrations and the 2005 toxic equivalency factors (TEFs) published by the World Health Organization (WHO) (van den Berg et al., 2006).

\section{Results and discussion}

\subsection{Concentrations of PCBs in sediments from the Murchison Bay}

Total PCB concentration ( $\left.\Sigma_{18} \mathrm{PCBs}\right)$ in Murchison Bay sediments varied widely with mean values ranging from 777 to $4325 \mathrm{pg} \mathrm{g}^{-1} \mathrm{dw}$ (Table 1). They were 3-fold higher than those reported in sediments from the Napoleon Gulf and Thurston Bay of Lake Victoria (Ssebugere et al., 2013b; Ssebugere et al., 2014). The PCB concentrations in the present study were comparable to data from other fresh water bodies in Africa: the Nile River in Egypt (El-Kady et al., 2007), Ghar El Melh lagoon in Tunisia (Ameur et al., 2011), Pangani River basin in Tanzania
(Hellar-Kihampa et al., 2013) and Congo River basin in the Democratic Republic of Congo (Verhaert et al., 2013). The results were also in the range of data found in sediments from tropical areas in Asia: the Mekong River delta in Vietnam (Carvalho et al., 2008) and riverine and coastal waters of Surabaya in Indonesia (Ilyas et al., 2011). The levels were lower compared to concentrations reported for industrialized areas in Europe and United States of America (Covaci et al., 2005; Vane et al., 2007; Vives et al., 2007; Castells et al., 2008; Jiao et al., 2009; Martinez et al., 2010; Mohammed et al., 2011) (Table 2).

The proportion of the 6 indicator PCBs ( $\Sigma_{6} \mathrm{PCBs}$ ) to $\Sigma_{18} \mathrm{PCBs}$ in this study were between 80 and $88 \%$ (with a mean value of $84 \%$ ). CB 138 and 153 were the predominant congeners, collectively accounting for $21 \%$ of the $\Sigma_{18}$ PCBs on average (Fig. 2). Barakat et al. (2012) also reported the predominance of CB 138 and CB 153 in surface sediments from Lake Maryut in Egypt. The accumulation of PCBs in the environment is directly linked to their degree of chlorination, stereochemistry and lipophilicity (Mazet et al., 2005). PCBs with a high degree of chlorination

Table 3

Mean concentrations and ranges ( $\mathrm{pg} \mathrm{g}^{-1}$ wet weight) of PCBs in fish species from the Murchison Bay.

\begin{tabular}{|c|c|c|c|c|c|c|c|c|}
\hline \multirow[b]{3}{*}{ Lipid content (\%) } & \multicolumn{2}{|l|}{ Station 1} & \multicolumn{2}{|l|}{ Station 2} & \multicolumn{2}{|l|}{ Station 3} & \multicolumn{2}{|l|}{ Station 4} \\
\hline & L. niloticus & O. niloticus & L. niloticus & O. niloticus & L. niloticus & O. niloticus & L. niloticus & O. niloticus \\
\hline & 2.8 & 1.5 & 2.3 & 1.4 & 2.2 & 1.1 & 1.6 & 1.2 \\
\hline \multicolumn{9}{|l|}{ Congener } \\
\hline CB 28 & $\begin{array}{l}38 \pm 22 \\
13-53\end{array}$ & $\begin{array}{l}14 \pm 7.0 \\
6.0-19\end{array}$ & $\begin{array}{l}62 \pm 21 \\
40-83\end{array}$ & $\begin{array}{l}13 \pm 3.6 \\
8.4-15\end{array}$ & $\begin{array}{l}23 \pm 7.5 \\
18-31\end{array}$ & $\begin{array}{l}32 \pm 14 \\
12-68\end{array}$ & $\begin{array}{l}26 \pm 15 \\
10-41\end{array}$ & $\begin{array}{l}9.3 \pm 0.7 \\
8.6-10\end{array}$ \\
\hline CB 52 & $\begin{array}{l}39 \pm 23 \\
23-65\end{array}$ & $\begin{array}{l}30 \pm 24 \\
7.8-55\end{array}$ & $\begin{array}{l}60 \pm 52 \\
10-128\end{array}$ & $\begin{array}{l}22 \pm 18 \\
9.0-43\end{array}$ & $\begin{array}{l}19 \pm 8.2 \\
10-27\end{array}$ & $\begin{array}{l}13 \pm 4.6 \\
10-18\end{array}$ & $\begin{array}{l}12 \pm 3.2 \\
9.6-16\end{array}$ & $\begin{array}{l}8.1 \pm 2.1 \\
5.6-9.5\end{array}$ \\
\hline СВ 101 & $\begin{array}{l}58 \pm 11 \\
45-66\end{array}$ & $\begin{array}{l}23 \pm 18 \\
2.1-37\end{array}$ & $\begin{array}{l}86 \pm 39 \\
50-128\end{array}$ & $\begin{array}{l}17 \pm 4.9 \\
12-22\end{array}$ & $\begin{array}{l}50 \pm 25 \\
22-65\end{array}$ & $\begin{array}{l}13 \pm 2.6 \\
10-15\end{array}$ & $\begin{array}{l}21 \pm 6.5 \\
16-28\end{array}$ & $\begin{array}{l}9.8 \\
4.9-14\end{array}$ \\
\hline CB 138 & $\begin{array}{l}183 \pm 98 \\
72-259\end{array}$ & $\begin{array}{l}43 \pm 9.9 \\
32-51\end{array}$ & $\begin{array}{l}84 \pm 38 \\
52-127\end{array}$ & $\begin{array}{l}17 \pm 13 \\
7.7-32\end{array}$ & $\begin{array}{l}61 \pm 31 \\
25-81\end{array}$ & $\begin{array}{l}10 \pm 1.3 \\
9.8-12\end{array}$ & $\begin{array}{l}62 \pm 32 \\
33-96\end{array}$ & $\begin{array}{l}18 \pm 13 \\
5.9-31\end{array}$ \\
\hline CВ 153 & $\begin{array}{l}220 \pm 58 \\
75-386\end{array}$ & $\begin{array}{l}37 \pm 13 \\
22-49\end{array}$ & $\begin{array}{l}98 \pm 55 \\
51-158\end{array}$ & $\begin{array}{l}22 \pm 14 \\
13-39\end{array}$ & $\begin{array}{l}76 \pm 43 \\
30-112\end{array}$ & $\begin{array}{l}12 \pm 2.1 \\
10-14\end{array}$ & $\begin{array}{l}72 \pm 15 \\
37-133\end{array}$ & $\begin{array}{l}18 \pm 12 \\
4.9-30\end{array}$ \\
\hline CB 180 & $\begin{array}{l}98 \pm 57 \\
37-151\end{array}$ & $\begin{array}{l}12 \pm 10 \\
5.1-24\end{array}$ & $\begin{array}{l}8.3 \pm 2.2 \\
\text { n.d. }-9.8\end{array}$ & $\begin{array}{l}3.8 \pm 2.5 \\
1.1-7.7\end{array}$ & $\begin{array}{l}13 \pm 6.7 \\
8-21\end{array}$ & $\begin{array}{l}3.2 \pm 1.4 \\
1.7-4.5\end{array}$ & $\begin{array}{l}11 \pm 6.7 \\
6.0-18\end{array}$ & $\begin{array}{l}4.9 \pm 3.0 \\
1.5-7.3\end{array}$ \\
\hline$\Sigma$ indicator-PCBs & $\begin{array}{l}636 \\
363-904\end{array}$ & $\begin{array}{l}159 \\
75-228\end{array}$ & $\begin{array}{l}398 \\
288-573\end{array}$ & $\begin{array}{l}95 \\
57-124\end{array}$ & $\begin{array}{l}242 \\
144-307\end{array}$ & $\begin{array}{l}83 \\
60-122\end{array}$ & $\begin{array}{l}204 \\
112-322\end{array}$ & $\begin{array}{l}68 \\
32-100\end{array}$ \\
\hline CB 77 & $\begin{array}{l}5.5 \pm 1.7 \\
3.8-7.1\end{array}$ & $\begin{array}{l}3.1 \pm 2.5 \\
1.2-5.9\end{array}$ & n.d. & $\begin{array}{l}1.0 \pm 0.4 \\
0.6-1.4\end{array}$ & $\begin{array}{l}2.2 \pm 0.9 \\
1.3-3.1\end{array}$ & $\begin{array}{l}1.3 \pm 0.2 \\
1.0-1.4\end{array}$ & $\begin{array}{l}1.3 \pm 0.1 \\
\text { n.d. }-1.4\end{array}$ & $\begin{array}{l}0.7 \\
\text { n.d.-0.7 }\end{array}$ \\
\hline CB 81 & $\begin{array}{l}0.9 \\
\text { n.d.-0.9 }\end{array}$ & $\begin{array}{l}1.1 \pm 0.6 \\
\text { n.d. }-1.5\end{array}$ & n.d. & n.d. & $\begin{array}{l}0.9 \pm 0.2 \\
\text { n.d. }-1.5\end{array}$ & n.d. & $\begin{array}{l}0.5 \pm 0.4 \\
\text { n.d. }-0.8\end{array}$ & $\begin{array}{l}1.1 \pm 0.1 \\
\text { n.d. }-1.1\end{array}$ \\
\hline CB 126 & $\begin{array}{l}1.0 \pm 0.7 \\
0.5-1.5\end{array}$ & n.d. & $\begin{array}{l}0.2 \\
\text { n.d.- }-0.2\end{array}$ & n.d. & n.d. & n.d. & $\begin{array}{l}0.8 \\
\text { n.d.- } 0.8\end{array}$ & n.d. \\
\hline CВ 169 & n.d. & $\begin{array}{l}0.2 \\
\text { n.d.- } 0.2\end{array}$ & n.d. & n.d & n.d. & n.d. & $\begin{array}{l}0.2 \\
\text { n.d. }-0.2\end{array}$ & $\begin{array}{l}0.1 \\
\text { n.d.-0.1 }\end{array}$ \\
\hline$\Sigma$ non-ortho PCBs & $\begin{array}{l}7.4 \\
5.7-7.6\end{array}$ & $\begin{array}{l}4.4 \\
2.4-6.6\end{array}$ & $\begin{array}{l}0.2 \\
\text { n.d.-0.2 }\end{array}$ & $\begin{array}{l}1.0 \\
0.6-1.4\end{array}$ & $\begin{array}{l}3.1 \\
2.4-3.1\end{array}$ & $\begin{array}{l}1.3 \\
1.0-1.4\end{array}$ & $\begin{array}{l}2.8 \\
\text { n.d.-3.2 }\end{array}$ & $\begin{array}{l}1.9 \\
0.7-1.1\end{array}$ \\
\hline СВ 105 & $\begin{array}{l}23 \pm 11 \\
13-34\end{array}$ & $\begin{array}{l}7.8 \pm 1.8 \\
5.8-8.9\end{array}$ & $\begin{array}{l}1.8 \pm 0.4 \\
1.4-2.2\end{array}$ & $\begin{array}{l}1.9 \pm 0.3 \\
1.7-2.2\end{array}$ & $\begin{array}{l}3.6 \pm 0.1 \\
3.5-3.7\end{array}$ & $\begin{array}{l}1.8 \pm 0.8 \\
1.0-2.6\end{array}$ & $\begin{array}{l}6.7 \pm 5.3 \\
1.5-12\end{array}$ & $\begin{array}{l}1.8 \pm 0.3 \\
1.4-2.1\end{array}$ \\
\hline CB 114 & $\begin{array}{l}1.5 \pm 0.8 \\
0.8-2.4\end{array}$ & $\begin{array}{l}1.6 \pm 0.7 \\
0.5-3.6\end{array}$ & $\begin{array}{l}4.2 \\
\text { n.d. }-4.2\end{array}$ & n.d. & $\begin{array}{l}0.2 \pm 0.1 \\
\text { n.d. }-0.2\end{array}$ & $\begin{array}{l}0.2 \\
\text { n.d.-0.2 }\end{array}$ & $\begin{array}{l}0.7 \pm 0.2 \\
\text { n.d. }-0.9\end{array}$ & $\begin{array}{l}0.3 \\
\text { n.d. }-0.3\end{array}$ \\
\hline CB 118 & $\begin{array}{l}80 \pm 47 \\
35-128\end{array}$ & $\begin{array}{l}47 \pm 13 \\
19-97\end{array}$ & $\begin{array}{l}23 \pm 16 \\
14-41\end{array}$ & $\begin{array}{l}6.5 \pm 1.2 \\
5.5-7.8\end{array}$ & $\begin{array}{l}17 \pm 3.1 \\
13-19\end{array}$ & $\begin{array}{l}6.0 \pm 1.6 \\
4.2-7.2\end{array}$ & $\begin{array}{l}25 \pm 19 \\
7.5-46\end{array}$ & $\begin{array}{l}5.5 \pm 1.9 \\
3.3-6.8\end{array}$ \\
\hline CB 123 & $\begin{array}{l}14 \pm 8.7 \\
4.4-22\end{array}$ & $\begin{array}{l}5.9 \pm 2.2 \\
2.7-12\end{array}$ & $\begin{array}{l}3.3 \pm 2.3 \\
1.4-5.8\end{array}$ & $\begin{array}{l}0.8 \pm 0.3 \\
\text { n.d. }-1.0\end{array}$ & $\begin{array}{l}2.3 \pm 0.5 \\
1.8-2.7\end{array}$ & $\begin{array}{l}0.7 \pm 0.3 \\
0.3-0.9\end{array}$ & $\begin{array}{l}4.3 \pm 3.9 \\
0.8-8.5\end{array}$ & $\begin{array}{l}0.7 \\
0.4-1.0\end{array}$ \\
\hline СВ 156 & $\begin{array}{l}8.5 \pm 4.7 \\
3.3-12\end{array}$ & $\begin{array}{l}2.3 \pm 0.2 \\
2.1-2.5\end{array}$ & $\begin{array}{l}2.8 \pm 2.1 \\
1.0-5.4\end{array}$ & $\begin{array}{l}0.8 \pm 0.5 \\
0.5-1.4\end{array}$ & $\begin{array}{l}2.3 \pm 0.8 \\
1.5-3.0\end{array}$ & $\begin{array}{l}0.6 \pm 0.1 \\
\text { n.d. }-0.6\end{array}$ & $\begin{array}{l}3.3 \pm 2.4 \\
1.9-6.1\end{array}$ & $\begin{array}{l}0.7 \pm 0.3 \\
\text { n.d. }-1.0\end{array}$ \\
\hline CВ 157 & $\begin{array}{l}1.7 \pm 1.3 \\
0.5-3.0\end{array}$ & $\begin{array}{l}0.4 \pm 0.1 \\
0.3-0.4\end{array}$ & n.d & $\begin{array}{l}0.1 \\
\text { n.d.- } 0.1\end{array}$ & $\begin{array}{l}1.8 \pm 0.4 \\
0.3-2.9\end{array}$ & $\begin{array}{l}0.1 \\
\text { n.d. }-0.1\end{array}$ & $\begin{array}{l}1.0 \pm 0.6 \\
\text { n.d. }-1.4\end{array}$ & $\begin{array}{l}0.6 \\
\text { n.d.-1.1 }\end{array}$ \\
\hline CB 167 & $\begin{array}{l}5.1 \pm 3.7 \\
1.3-8.6\end{array}$ & $\begin{array}{l}0.7 \pm 0.2 \\
0.6-0.9\end{array}$ & $\begin{array}{l}2.8 \pm 1.7 \\
1.2-5.9\end{array}$ & $\begin{array}{l}0.3 \pm 0.2 \\
\text { n.d. }-0.5\end{array}$ & $\begin{array}{l}1.2 \pm 0.4 \\
0.5-2.0\end{array}$ & $\begin{array}{l}0.3 \pm 0.1 \\
\text { n.d. }-0.4\end{array}$ & $\begin{array}{l}1.6 \pm 1.2 \\
0.4-2.8\end{array}$ & $\begin{array}{l}0.6 \\
\text { n.d.- }-0.6\end{array}$ \\
\hline CB 189 & $\begin{array}{l}2.5 \pm 0.3 \\
\text { n.d. }-2.8\end{array}$ & $\begin{array}{l}1.0 \pm 0.7 \\
\text { n.d. }-1.5\end{array}$ & $\begin{array}{l}3.1 \\
\text { n.d.-3.1 }\end{array}$ & n.d. & $\begin{array}{l}0.5 \\
\text { n.d. }-0.5\end{array}$ & $\begin{array}{l}0.1 \\
\text { n.d.- } 0.1\end{array}$ & $\begin{array}{l}1.4 \\
\text { n.d.-1.4 }\end{array}$ & n.d. \\
\hline$\Sigma$ mono-ortho PCBs & $\begin{array}{l}136 \\
58-213\end{array}$ & $\begin{array}{l}66 \\
34-123\end{array}$ & $\begin{array}{l}41 \\
20-67\end{array}$ & $\begin{array}{l}10 \\
7.8-12\end{array}$ & $\begin{array}{l}29 \\
21-34\end{array}$ & $\begin{array}{l}9.7 \\
6.1-12\end{array}$ & $\begin{array}{l}44 \\
12-80\end{array}$ & $\begin{array}{l}10 \\
5.9-12\end{array}$ \\
\hline$\Sigma_{12} \mathrm{dl}-\mathrm{PCBs}$ & $\begin{array}{l}143 \\
66-219\end{array}$ & $\begin{array}{l}71 \\
37-126\end{array}$ & $\begin{array}{l}41 \\
20-67\end{array}$ & $\begin{array}{l}11 \\
9.0-13\end{array}$ & $\begin{array}{l}32 \\
24-37\end{array}$ & $\begin{array}{l}11 \\
7.1-11\end{array}$ & $\begin{array}{l}47 \\
12-83\end{array}$ & $\begin{array}{l}12 \\
7.0-13\end{array}$ \\
\hline$\Sigma_{18} \mathrm{PCBs}$ & $\begin{array}{l}779 \\
429-1123\end{array}$ & $\begin{array}{l}229 \\
201-275\end{array}$ & $\begin{array}{l}439 \\
308-641\end{array}$ & $\begin{array}{l}106 \\
66-137\end{array}$ & $\begin{array}{l}274 \\
168-343\end{array}$ & $\begin{array}{l}93 \\
72-135\end{array}$ & $\begin{array}{l}251 \\
124-404\end{array}$ & $\begin{array}{l}80 \\
39-111\end{array}$ \\
\hline $\mathrm{WHO}_{2005}$-TEQs & $\begin{array}{l}0.16 \\
0.07-0.22\end{array}$ & $\begin{array}{l}0.03 \\
0.02-0.03\end{array}$ & $\begin{array}{l}0.15 \\
0.01-0.39\end{array}$ & $\begin{array}{l}0.06 \\
0.01-0.15\end{array}$ & $\begin{array}{l}0.10 \\
0.03-0.20\end{array}$ & $\begin{array}{l}0.04 \\
0.02-0.07\end{array}$ & $\begin{array}{l}0.07 \\
0.04-0.09\end{array}$ & $\begin{array}{l}0.06 \\
0.02-0.11\end{array}$ \\
\hline
\end{tabular}

The number of fish muscle homogenates per species per station $(\mathrm{N})=3$; n.d. - non-detectable. 
(such as CB 138 and CB 153) have lower chemical degradation rates than the less chlorinated PCBs and thus are retained in aquatic systems to a greater degree, where they may bioaccumulate in organisms like fish (Lavandier et al., 2013).

The PCB levels at station 1 were significantly higher $(p<0.05$, Mann-Whitney $U$-test) than those from stations 3 and 4 . However, statistical analysis between stations 1 and 2 showed no significant difference between the two locations. Station 1 is located near the mouth of the Nakivubo channel which brings in effluents from a number of chemical manufacturing factories, industrial waste treatment plants and municipal solid waste incinerators. Furthermore, in close vicinity of the station 1 are industrial facilities and Port bell where ships anchor on their way to and from Ports like Mwanza in Tanzania, Kisumu in Kenya and Jinja Port in Uganda. Although, the shipping industry promotes economic development in the area, it inevitably brings pollution related problems.

High concentrations of PCBs were also observed at station 2 (mean value of the $\Sigma_{18}$ PCBs in sediments was 2576, range $1753-3970 \mathrm{pg} \mathrm{g}^{-1} \mathrm{dw}$ ). Station 2 is situated close to the Ggaba landing site and a local market. Besides the market a lot of commercial, domestic and industrial activities occur near the station and could be releasing domestically-produced commercial PCB mixtures into the lake. The PCB levels in sediments from the outer part of the Murchison Bay (stations 3 and 4), both far from industrial complexes, were an order of magnitude lower compared to those in the inner Bay (Fig. 3). The low PCB levels in the outer Murchison Bay are likely due to the far offshore distance from the pollution sources.

The $\mathrm{WHO}_{2005}$-TEQs for the $12 \mathrm{dl}$-PCBs in sediments of the present study varied from 0.04 to $0.64 \mathrm{pg} \mathrm{TEQ} \mathrm{g}^{-1}$. Station 1 had the highest TEQ values ranging from 0.37 to $0.64 \mathrm{pg}^{\mathrm{T}} \mathrm{TEQ} \mathrm{g}^{-1}$ (mean $0.55 \mathrm{pg} \mathrm{TEQ}^{-1}$ ) while station 4 had the lowest (range 0.07 to 0.14 , mean $0.10 \mathrm{pg}$ TEQ $^{-1}$ ). CB 126 was the highest contributor to the $\Sigma_{12}$ TEQs (70-89\%) compared to other congeners at all locations. The $\mathrm{WHO}_{2005}$-TEQs in most samples from the Murchison Bay were below

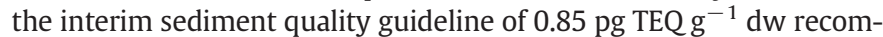
mended by the Canadian Council of Ministers of the Environment (2002). Thus the dl-PCB concentrations in the present work are unlikely to pose a threat to benthic organisms.

\subsection{Concentrations of PCBs in fish from the Murchison Bay}

Table 3 presents the PCB concentrations in fish muscle homogenates of $L$. niloticus and $O$. niloticus. Average concentrations of the $\Sigma_{18}$ PCBs ranged from 80 to $779 \mathrm{pg} \mathrm{g}^{-1}$ wet weight (ww). The concentrations were in the same range as those reported in similar fish species from the Napoleon Gulf of Lake Victoria (94-716 $\mathrm{pg} \mathrm{g}^{-1}$ ww: Ssebugere et al., 2013a; Ssebugere et al., 2014) and River Nile in Egypt (695-853 pg ${ }^{-1}$ ww; El-Kady et al., 2007). In neighboring Burundi, Manirakiza et al. (2002) reported PCB concentrations between 44900 and $166700 \mathrm{pg} \mathrm{g}^{-1} \mathrm{lw}$ in 0 . niloticus collected from Lake Tanganyika.
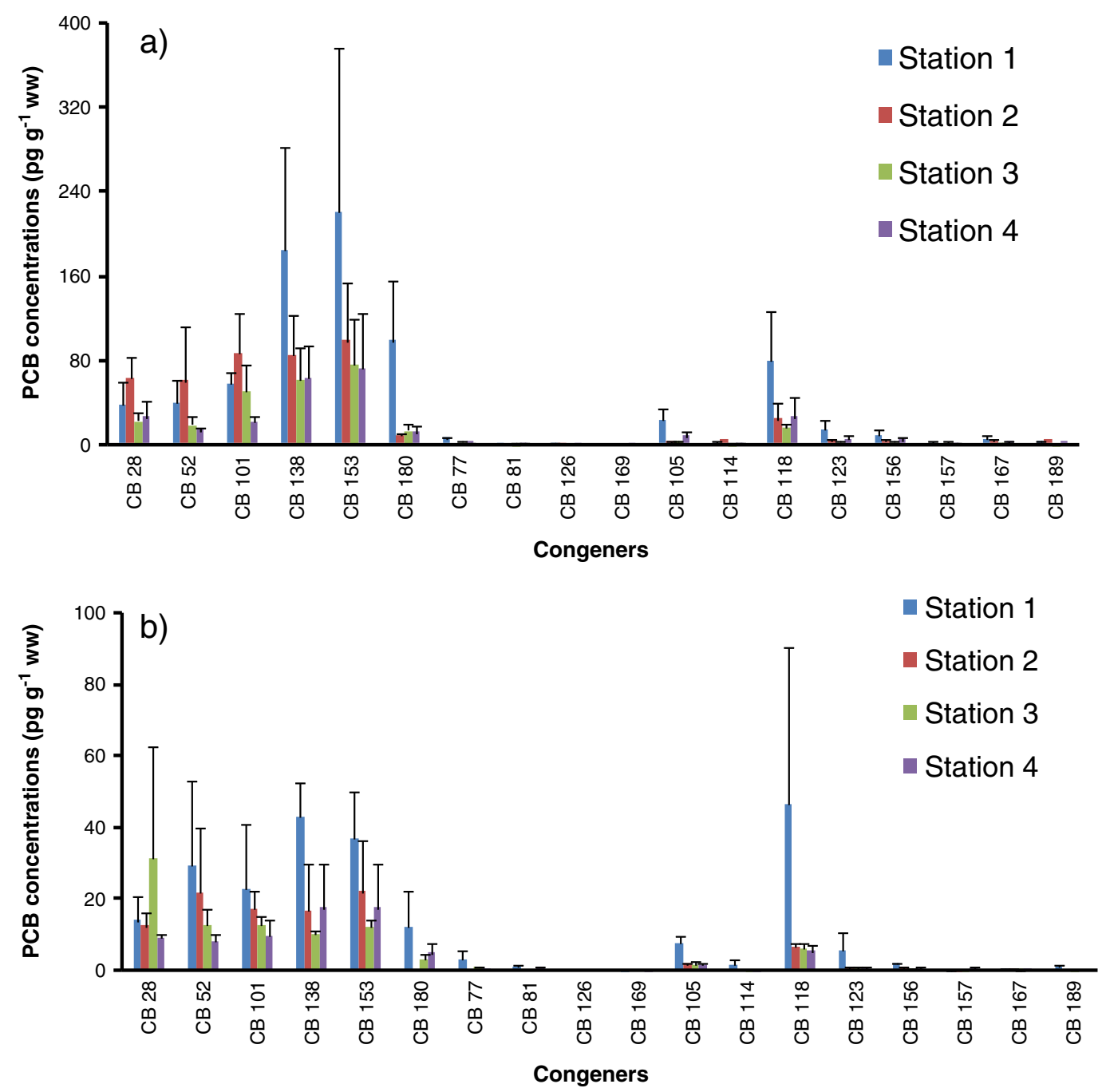

Fig. 4. Mean PCB concentrations in fish per station. a) Mean PCB concentrations in L. niloticus per station. b) Mean PCB concentrations in O. niloticus per station. 
These levels were at least an order of magnitude higher compared to the present results. In Egypt, Said et al. (2008) reported PCB concentrations varying from 3320 to $72060 \mathrm{pg} \mathrm{g}^{-1} \mathrm{lw}$ in fish (O. niloticus and Clarias gariepinus) from Lake Burullus. The concentrations were again higher than the data in the study herein. Studies outside Africa have also reported PCBs in fish. In Hawai'i, PCB levels ranging from 51900 to $89420 \mathrm{pg} \mathrm{g}^{-1}$ lw were reported in tilapia (Oreochromis mossambicus) collected from the Manoa stream and Ala Wai Canal of O'ahu (Yang et al., 2008). The PCB levels were also higher than those reported in this study. In South China, Nie et al. (2006) reported PCB concentrations ranging from 5150 to $226000 \mathrm{pg} \mathrm{g}^{-1} \mathrm{lw}$ in tilapia (Tilapia mossambica) from Pearl River Delta. The concentrations are still higher compared to the data in the current study.

The $\Sigma_{18} \mathrm{PCBs}$ in L. niloticus at station 1 was one-fold higher than that at station 2 and about three times higher than that at station 4 . The $\Sigma_{18}$ PCBs at stations 3 and 4 were quite similar. For O. niloticus, still station 1 showed the highest levels of the $\Sigma_{18} \mathrm{PCBs}$ (mean value $229 \mathrm{pg} \mathrm{g}^{-1}$ ) compared to other stations. Station 1 was followed by station 2 and then station 3, while station 4 had the lowest concentration. The concentrations of the different $\mathrm{PCB}$ congeners at the different stations for the 2 fish species are shown in Fig. 4.

The PCB concentrations in L. niloticus were markedly higher ( $p<0.05$, Mann-Whitney $U$-test) than those in 0 . niloticus at all study sites. The nature of the diet could be a possible explanation for such an inter-specific differences in concentrations. L. niloticus are exclusively piscivorous, consuming all available fish species including its own siblings whereas 0 . niloticus relies on zooplankton and macroinvertebrates (Ogari, 1984; Njiru et al., 2004). Earlier studies established that PCBs can have great variations among different fish, as well as within species depending on their feeding habits and trophic levels (Suedel et al., 1994; Vander Zanden and Rasmussen, 1996; Davis et al., 2002; van der Oost et al., 2003).

On the whole, the $\Sigma_{6}$ indicator PCBs in fish of the present study were an order of magnitude higher than the $\Sigma_{7}$ mono-ortho-PCBs and $\Sigma_{4}$ nonortho-PCBs. However, the $\Sigma_{6}$ indicator PCB levels (83-636 $\mathrm{pg} \mathrm{g}^{-1}$ ) in this study were within the limit of $75000 \mathrm{pg} \mathrm{g}^{-1}$ ww set for fish by the European Commission (EC, 2011), implying that the study fish were fit for human consumption in regard to indicator PCBs. Indicator PCB congeners such as CB 138 and CB 153 which were predominant in the sediments were also major contributors in L. niloticus (Fig. 5). However, variations in contribution of the indicator PCBs to the $\Sigma_{18} \mathrm{PCBs}$ were observed for L. niloticus at the different stations. For the coplanar PCBs, the mono-ortho PCBs were the predominant congeners in most fish (their contribution to the $\Sigma_{12}$ PCBs ranged from 84 to $100 \%$ ). CB 118 was the most abundant congener, accounting for $46-69 \%$ of the $\Sigma_{12} \mathrm{PCBs}$, in comparison to other coplanar compounds. The high levels of CB 118 in the two fish species could be due to the inability of the species to metabolize the congener after accumulation. The presence of $\mathrm{CB}$ 118 in our samples suggests paint additives, municipal waste plants, iron ore sintering plants or medical waste incinerators as potential sources of PCBs (Abad et al., 2006; Aries et al., 2006; Shin et al., 2006; Chen et al., 2009; Jartun et al., 2009). Furthermore, it is likely that the
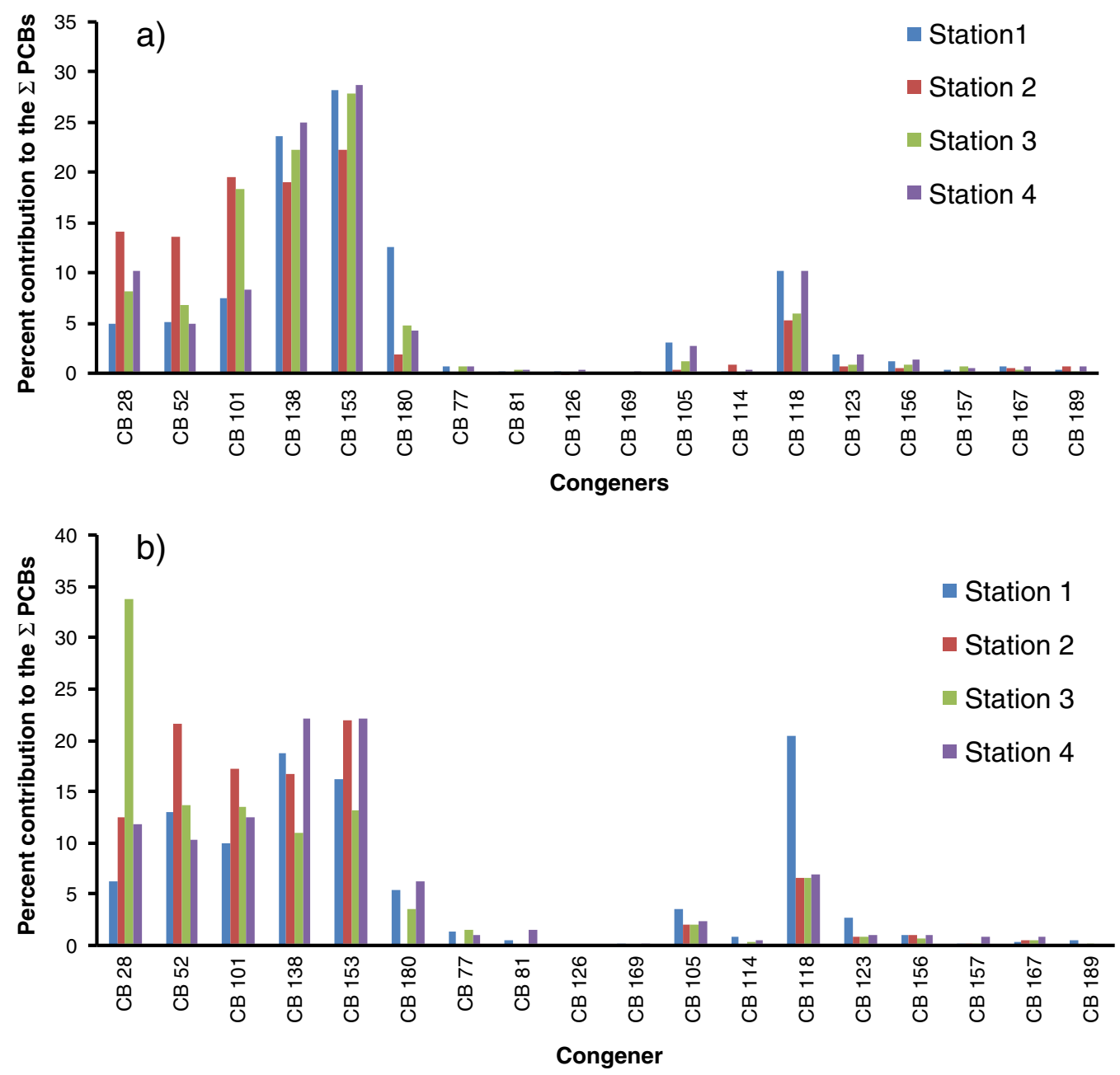

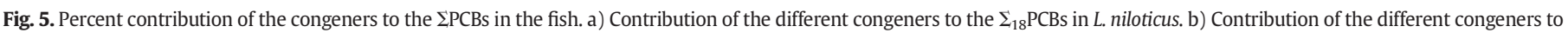
the $\Sigma_{18} \mathrm{PCBs}$ in 0 . niloticus. 
PCBs came from the residues of former applications like transformers, electrical equipment and other industrial uses. The use of these applications containing PCBs was common in Uganda before their commercial production was banned worldwide in 1979 (National Environment Management Authority, 2007).

The $\mathrm{WHO}_{2005}$-TEQs for dl-PCBs in the study herein were 0.01-0.39 and $0.01-0.11 \mathrm{pg} \mathrm{g}^{-1} \mathrm{ww}$ for L. niloticus and 0 . niloticus, respectively. The non-ortho PCBs exhibited the highest contribution to the $\Sigma_{12}$ PCBs (>75\%) compared to the mono-ortho PCBs in both fish species. A similar observation was also reported by Ssebugere et al. (2013a) in fish from the Napoleon Gulf and Thurston Bay of Lake Victoria. The $\mathrm{WHO}_{2005}$-TEQs in the present study were within the permissible level of $3.5 \mathrm{pg} \mathrm{g}^{-1}$ ww set by the European Commission (EC, 2011), suggesting that the fish from the Murchison Bay were safe for consumption with respect to dl-PCBs.

\section{Conclusions}

To our knowledge this is the first study to record PCB concentrations in sediments and fish from the Murchison Bay. The station closest to Nakivubo channel had the highest PCB concentrations compared to other locations. Land based activities such as urban centers, commercial and industrial establishments are likely the major sources of PCB contamination. The PCB concentrations in the present study were generally comparable to those of most studies in Africa but lower than the data examined elsewhere in the world. The WHO-TEQs were below the recommended values set for sediments and fish, indicating that the risk posed by PCBs is limited, compared to other health and environmental issues, such as the high disease burden due to diarrhea and other infectious diseases, associated with a limited availability of sanitation and treated water (Yeka et al., 2012; Tumwebaze et al., 2013; McElligott et al., 2013; Bwire et al. 2013; Katukiza et al., 2014). However, regulatory controls for monitoring and mitigating wastewater emissions into Lake Victoria need to be implemented and emphasized by the relevant governmental agencies in Uganda.

\section{Conflict of interest}

\section{There is no conflict of interest.}

\section{Acknowledgments}

Patrick Ssebugere is grateful for the research fellowship by the Third World Academy of Sciences (TWAS) and Chinese Academy of Sciences (CAS). The authors would like acknowledge the financial support from the Chinese Academy of Sciences (YSW2013B01) and National Natural Science Foundation of China (41276195, 21277165 and 21107121).

\section{References}

Abad E, Martinez K, Caixach J, Rivera J. Polychlorinated dibenzo-p-dioxins, dibenzofurans and 'dioxin-like' PCBs in flue gas emissions from municipal waste management plants. Chemosphere 2006;63:570-80.

Åberg A, Maclead M, Wiberg K. Physical-chemical property data for dibenzo-p-dioxins (DD), dibenzofuran (DF), and chlorinated DD/Fs: a critical review and recommended values. J Phys Chem Ref Data 2008;37:1997-2008.

Åberg A, Tysklind M, Nilsson T, MacLeod M, Hanberg A, Andersson R, et al. Exposure assessment at a PCDD/F contaminated site in Sweden-field measurements of exposure media and blood serum analysis. Environ Sci Pollut Res 2010;17:26-39.

Adu-Kumi S, Kawano M, Shiki Y, Yeboah PO, Carboo D, Pwamang J, et al. Organochlorine pesticides (OCPs), dioxin-like polychlorinated biphenyls (dl-PCBs), polychlorinated dibenzo-p-dioxins and polychlorinated dibenzo furans (PCDD/Fs) in edible fish from Lake Volta, Lake Bosumtwi and Weija Lake in Ghana. Chemosphere 2010; 81(6):675-84.

Ameur WB, Trabelsi S, El Bedoui B, Driss MR. Polychlorinated biphenyls in sediments from Ghar El Melh lagoon,Tunisia. Bull Environ Contam Toxicol 2011;86(5):539-44.

Aries E, Anderson DR, Fisher R, Fray TAT, Hemfrey D. PCDD/F and "dioxin-like" PCB emissions from iron ore sintering plants in the UK. Chemosphere 2006;65:1470-80.
Banadda EN, Kansiime F, Kigobe M, Kizza M, Nhapi I. Land use based non-point source pollution: a threat to Murchison bay water quality in Uganda. Water Qual II Suppl 2009;1:93-104.

Banadda N, Ayaa F, Nhapi I, Wali UG, Kimwaga RJ, Mashauri DA. Modeling diffusive flux of non point source pollutants in Lake Victoria: a comparison study of Fick's law and the Fokker-Planck law. Open Environ Eng J 2011a;4:105-11.

Banadda N, Nhapi I, Wali UG. Determining and modeling the dispersion of non point source pollutants in Lake Victoria: a case study of Ggaba landing site in Uganda. Afr J Environ Sci Technol 2011b;5(3):178-85.

Barakat AO, Mostafa A, Wade TL, Sweet ST, El Sayed NB. Spatial distribution and temporal trends of persistent organochlorine pollutants in sediments from Lake Maryut, Alexandria, Egypt. Mar Pollut Bull 2012;64(2):395-404.

Bwire G, Malimbo M, Maskery B, Kim EY, Mogasale V, Levin A. The burden of cholera in Uganda. PLoS Negl Trop Dis 2013;7(12):e2545.

Canadian Council of Ministers of the Environment. Canadian environmental quality guidelines for polychlorinated dibenzo-p-dioxins and polychlorinated dibenzofurans (PCDD/Fs). Technical supporting document. Ottawa: National Guidelines and Standards Office, Environmental Quality Branch; 2002.

Carvalho FP, Villeneuve JP, Cattini C, Tolosa I, Thuan DD, Nhan DD. Agrochemical and polychlorobyphenyl (PCB) residues in the Mekong River delta, Vietnam. Mar Pollut Bull 2008;56(8):1476-85.

Castells P, Parera J, Santos FJ, Galceran MT. Occurrence of polychlorinated naphthalenes, polychlorinated biphenyls and short-chain chlorinated paraffins in marine sediments from Barcelona (Spain). Chemosphere 2008;70(9):1552-62.

Chen T, Li XD, Yan JH, Jin YQ. Polychlorinated biphenyls emission from a medical waste incinerator in China. J Hazard Mater 2009;172:1339-43.

Covaci A, Gheorghe A, Voorspoels A, Maervoet J, Redeker ES, Blust R, et al. Polybrominated diphenyl ethers, polychlorinated biphenyls and organochlorine pesticides in sediment cores from Western Scheldt River (Belgium): analytical aspects and depth profiles. Environ Int 2005;31:367-75.

Darko G, Akoto O, Oppong C. Persistent organochlorine pesticide residues in fish sediments and water from Lake Bosomtwi, Ghana. Chemosphere 2008;72(1):21-4.

Davis JA, May MD, Greenfield BK, Fairey R, Roberts C, Ichikawa G, et al. Contaminant concentrations in sport fish from San Francisco Bay, 1997. Mar Pollut Bull 2002;44: $1117-29$.

EC. Commission Regulation (EU) No 1259/2011 of 2 December 2011 amending regulation (EC) No 1881/2006 as regards maximum levels for dioxins, dioxin-like PCBs and non dioxin-like PCBs in foodstuffs. Off J Eur Union 2011:320/18-23.

El-Kady AA, Abdel-Wahhab MA, Henkelmann B, Belal MH, Morsi MK, Galal SM, et al. Polychlorinated biphenyl, polychlorinated dibenzo-p-dioxin and polychlorinated dibenzofuran residues in sediments and fish of the River Nile in the Cairo region. Chemosphere 2007;68(9):1660-8.

Eqani SA, Malik RN, Cincinelli A, Zhang G, Mohammad A, Qadir A, et al. Uptake of organochlorine pesticides (OCPs) and polychlorinated biphenyls (PCBs) by river water fish: the case of River Chenab. Sci Total Environ 2013;450-451:83-91.

Gidarakos E, Petrantonaki M, Anastasiadou K, Schramm KW. Characterization and hazard evaluation of bottom ash produced from incinerated hospital. J Hazard Mater Waste 2009;172(2-3):935-42.

Gustavson KE, Burton GA, Francingues NR, Reible DD, Vorhees DJ, Wolfe JR. Evaluating the effectiveness of contaminated-sediment dredging. Environ Sci Technol 2008;42: 5042-7.

Ha MH, Lee DH, Jacobs DR. Association between serum concentrations of persistent organic pollutants and self-reported cardiovascular disease prevalence. Results from the national health and nutrition examination survey, 1999-2002. Environ Health Perspect 2007;115:1204-9.

Ha MH, Lee DH, Son HK, Park SK, Jacobs DR. Association between serum concentrations of persistent organic pollutants and prevalence of newly diagnosed hypertension: results from the National Health and Nutrition Examination Survey 1999-2002. J Hum Hypertens 2009;23:274-86.

Haande S, Rohrlack T, Semyalo PR, Brettum P, Edvardsen B, Lyche-Solheim A, et al. Phytoplankton dynamics and cyanobacterial dominance in Murchison Bay of Lake Victoria (Uganda) in relation to environmental conditions. Limnologica 2011;41:20-9.

Hellar-Kihampa H, De Wael K, Lugwisha E, Malarvannan G, Covaci A, Van Grieken R. Spatial monitoring of organohalogen compounds in surface water and sediments of a rural-urban river basin in Tanzania. Sci Total Environ 2013;447:186-97.

Helm PA, Gewurtz SB, Whittle DM, Marvin CH, Fisk AT, Tomy GT. Occurrence and biomagnification of polychlorinated naphthalenes and non- and mono-ortho PCBs in Lake Ontario sediment and biota. Environ Sci Technol 2008;42(4):1024-31.

Huang X, Hites RA, Foran JA, Hamilton C, Knuth BA, Schwager SJ, et al. Consumption advisories for salmon based on risk of cancer and non cancer health effects. Environ Res 2006:101:263-74.

Ilyas M, Sudaryanto A, Setiawan IE, Riyadi AS, Isobe T, Takahashi S, et al. Characterization of polychlorinated biphenyls and brominated flame retardants in sediments from riverine and coastal waters of Surabaya, Indonesia. Mar Pollut Bull 2011: 62(1):89-98.

Jartun M, Ottesen RT, Steinnes E, Volden T. Painted surfaces-important sources of polychlorinated biphenyls (PCBs) contamination to the urban and marine environment. Environ Pollut 2009;157(1):295-302.

Jiao L, Zheng GJ, Minh TB, Richardson B, Chen L, Zhang Y, et al. Persistent toxic substances in remote lake and coastal sediments from Svalbard, Norwegian Arctic: levels, sources and fluxes. Environ Pollut 2009;157(4):1342-51.

Jönsson A, Gustafsson Ö, Axelman J, Sundberg H. Global accounting of PCBs in the continental shelf sediments. Environ Sci Technol 2003;37:245-55.

Katukiza AY, Ronteltap M, van der Steen P, Foppen JWA, Lens PNL. Quantification of microbial risks to human health caused by waterborne viruses and bacteria in an urban slum. J Appl Microbiol 2014;116(2):447-63. 
Lavandier R, Quinete N, Hauser-Davis RA, Dias PS, Taniguchi S, Montone R, et al. Polychlorinated biphenyls (PCBs) and polybrominated diphenyl ethers (PBDEs) in three fish species from an estuary in the southeastern coast of Brazil. Chemosphere 2013;90(9):2435-43.

Lee DH, Lee IK, Jin SH, Steffes M, Jacobs Jr DR. Association between serum concentrations of persistent organic pollutants and insulin resistance among nondiabetic adults: results from the national health and nutrition examination survey 1999-2002. Diabetes Care 2007a;30:622-8.

Lee DH, Lee IK, Porta M, Steffes M, Jacobs Jr DR. Relationship between serum concentrations of persistent organic pollutants and the prevalence of metabolic syndrome among non-diabetic adults: results from the National Health and Nutrition Examination Survey 1999-2002. Diabetologia 2007b;50:1841-51.

Lundin L, Gomez-Rico MF, Forsberg C, Nordenskjöld C, Jansson S. Reduction of PCDD, PCDF and PCB during co-combustion of biomass with waste products from pulp and paper industry. Chemosphere 2013;91:797-801.

Manirakiza P, Covaci A, Nizigiymana L, Ntakimazi G, Schepens P. Persistent chlorinated pesticides and polychlorinated biphenyls in selected fish species from Lake Tanganyika, Burundi, Africa. Environ Pollut 2002;117:447-55.

Martinez A, Norström K, Wang K, Hornbuckle CK. Polychlorinated biphenyls in the surficial sediment of Indiana Harbor and Ship Canal, Lake Michigan. Environ Int 2010; 36(8):849-54.

Mazet A, Keck G, Berny P. Concentrations of PCBs, organochlorine pesticides and heavy metals (lead, cadmium, and copper) in fish from the Drome river: potential effects on otters (Lutra lutra). Chemosphere 2005;61:810-6.

McElligott JT, Naaktgeboren C, Makuma-Massa H, Summer AP, Deal JL. Prevalence of intestinal protozoa in communities along the Lake Victoria region of Uganda. Int J Infect Dis 2013;17(8):e658-9.

Miller A, Hedman JE, Nyberg E, Haglund P, Cousins IT, Wiberg K, et al. Temporal trends in dioxins (polychlorinated dibenzo-p-dioxin and dibenzofurans) and dioxin-like polychlorinated biphenyls in Baltic herring (Clupea harengus). Mar Pollut Bull 2013; 73:220-30.

Mohammed A, Peterman P, Echols K, Feltz K, Tegerdine G, Manoo A, et al. Polychlorinated biphenyls (PCBs) and organochlorine pesticides (OCPs) in harbor sediments from Sea Lots, Port-of-Spain, Trinidad and Tobago. Mar Pollut Bull 2011;62(6):1324-32.

Moon HB, Choi M, Choi HG, Kannan K. Severe pollution of PCDD/Fs and dioxin-like PCBs in sediments from Lake Shihwa, Korea: tracking the source. Mar Pollut Bull 2012;64: 2357-63.

National Environment Management Authority. Identification and quantification of dioxins and furans in Uganda: POPs inventory report. Uganda: Kampala; 2007.

Nie PX, Lan YC, An CT, Wong HM, Li BK. Distributions and congener patterns of PCBs in fish from major aquaculture areas in the Pearl River Delta, South China. Hum Ecol Risk Assess 2006;12(2):363-73.

Njiru M, Okeyo-Owuor BJ, Muchiri M, Cowx GI. Shifts in the food of Nile tilapia, Oreochromis niloticus (L.) in Lake Victoria, Kenya. Afr J Ecol 2004;42:163-70.

Ogari J. Distribution, food and feeding habits of *Lates niloticus* in Nyanza Gulf of Lake Victoria (Kenya). FAO Fish Rep 1984;335:68-80.

Parnell PE, Groce AK, Stebbins TD, Dayton PK. Discriminating sources of PCB contamination in fish on the coastal shelf off San Diego, California (USA). Mar Pollut Bull 2008;56:1992-2002.

Said TO, El Moselhy KM, Rashad AA, Shreadah MA. Organochlorine contaminants in water, sediment and fish of Lake Burullus, Egyptian Mediterranean Sea. Bull Environ Contam Toxicol 2008;81(2):136-46.

Shin SK, Kim KS, You JC, Song BJ, Kim JG. Concentration and congener patterns of polychlorinated biphenyls in industrial and municipal waste incinerator flue gas, Korea. J Hazard Mater 2006;133:53-9.

Ssebugere P, Kiremire BT, Henkelmann B, Bernhöft S, Wasswa J, Kasozi GN, et al. PCDD/Fs and dioxin-like PCBs in surface sediments from Lake Victoria, East Africa. Sci Total Environ 2013a;454-455:528-33.

Ssebugere P, Kiremire TB, Henkelmann B, Bernhöft S, Kasozi GN, Wasswa J, Schramm K-W. PCDD/Fs and dioxin-like PCBs in fish species from Lake Victoria, East Africa. Chemosphere 2013b;92(3):317-21.
Ssebugere P, Sillanpää M, Kiremire TB, Gabriel Kasozi NK, Wang P, Samuel Sojinu OS, Otieno OP, Zhu N, Zhu C, Zhang H, Shang H, Ren D, Li Y, Zhang Q, Jiang G. Polychlorinated biphenyls and hexachlorocyclohexanes in sediments and fish species from the Napoleon Gulf of Lake Victoria, Uganda. Sci Total Environ 2014:481:55-60.

Suedel BC, Boraczek JA, Peddicord RK, Clifford PA, Dillon TM. Trophic transfer and biomagnification potential of contaminants in aquatic ecosystems. Rev Environ Contam Toxicol 1994:136:21-89.

Sundqvist KL, Tysklind M, Cato I, Bignert A, Wiberg K. Levels and homologue profiles of PCDD/Fs in sediments along the Swedish coast of the Baltic Sea. Environ Sci Pollut Res 2009a; 16:396-409.

Sundqvist KL, Tysklind M, Geladi P, Cato I, Wiberg K. Congener fingerprints of tetrathrough octa-chlorinated dibenzo-p-dioxins and dibenzofurnas in Baltic surface sediments and relations to potential sources. Chemosphere 2009b;77:612-20.

Tumwebaze IK, Orach CG, Niwagaba C, Luthi C, Mosler HJ. Sanitation facilities in Kampala slums, Uganda: users' satisfaction and determinant factors. Int J Environ Health Res 2013;23(3):191-204.

Uemura H, Arisawa K, Hiyoshi M, Kitayama A, Takami H, Sawachika F, et al. Prevalence of metabolic syndrome associated with body burden levels of dioxin and related compounds among Japan's general population. Environ Health Perspect 2009;117: 568-73.

Van den Berg M, Birnbaum LS, Denison M, De Vito M, Farland W, Feeley M, et al. The 2005 World Health Organization reevaluation of human and mammalian toxic equivalency factors for dioxins and dioxin-like compounds. Toxicol Sci 2006;93:223-41.

Van der Oost R, Beyer J, Vermeulen NPE. Fish bioaccumulation and biomarkers in environmental risk assessment: a review. Environ Toxicol Pharmacol 2003;13: 57-149.

Vander Zanden MJ, Rasmussen JB. A trophic position model of pelagic food webs: impact on contaminant bioaccumulation in lake trout. Ecol Monogr 1996:66:451-77.

Vane HC, Harrison I, Kim WA. Polycyclic aromatic hydrocarbons (PAHs) and polychlorinated biphenyls (PCBs) in sediments from the Mersey Estuary, U.K. Sci Tot Environ 2007;374:112-26.

Verhaert V, Covaci A, Bouillon S, Abrantes K, Musibono D, Bervoets L, et al. Baseline levels and trophic transfer of persistent organic pollutants in sediments and biota from the Congo River Basin (DR Congo). Environ Int 2013;59:290-302.

Vives I, Canuti E, Castro-Jiménez J, Christoph EH, Eisenreich SJ, Hanke G, et al. Occurrence of polychlorinated dibenzo-p-dioxins and dibenzofurans (PCDD/Fs), polychlorinated biphenyls (PCBs) and polybrominated diphenyl ethers (PBDEs) in Lake Maggiore (Italy and Switzerland). J Environ Monit 2007;9(6):589-98.

Wang P, Zhang H, Fu J, Li Y, Wang T, Wang Y, et al. Temporal trends of PCBs, PCDD/Fs and PBDEs in soils from an E-waste dismantling area in East China. Environ Sci Process Impacts 2013;15(10):1897-903.

Wasswa J. Characterization of selected pesticide residues in sediments of the Ugandan side of Lake Victoria. PhD Thesis Kampala, Uganda: Department of Chemistry, Makerere University; 2009.

Wasswa J, Kiremire BT, Nkedi-Kizza P, Mbabazi J, Ssebugere P. Organochlorine pesticide residues in sediments from the Ugandan side of Lake Victoria. Chemosphere 2010; 82(1):130-6.

Wiberg K, McLachlan M, Jonsson P, Johansson N, Josefsson S, Knekta E, et al. Sources, transport reservoirs and fate of dioxins, PCBs, and HCB in the Baltic Sea environment. Report No. 5912 for the Swedish Environmental Protection Agency 978-91-6205912-5; 2009.

Yang F, Wilcox B, Jin S, Alonso Aguirre A, Rougée L, Xu Y, Lu Y. Detection and quantitative analysis of polychlorinated biphenyls in tilapia from Hawaiian waters. Chemosphere 2008;73(1):133-7.

Yeka A, Gasasira A, Mpimbaza A, Achan J, Nankabirwa J, Nsobya S, et al. Malaria in Uganda: challenges to control on the long road to elimination: I. Epidemiology and current control efforts. Acta Trop 2012;121(3):184-95. 